\title{
Term Structure Models with Shot-noise Effects
}

\author{
Raquel M. Gaspar \\ Advance Reseach Center \\ ISEG, Technical University of Lisbon \\ e-mail: Rmgaspar@iseg.utl.pt
}

\author{
Thorsten Schmidt \\ Department of Mathematics \\ University of Leipzig \\ e-mail: thorsten.schmidt@math.uni-leipzig.de
}

WORKI NG PAPER N.3/ 2007

July 2007

\begin{abstract}
This work proposes term structure models consisting of two parts: a part which can be represented in exponential quadratic form and a shot noise part. These term structure models allow for explicit expressions of various derivatives. In particular, they are very well suited for credit risk models.

The goal of the paper is twofold. First, a number of key building blocks useful in term structure modelling are derived in closed-form. Second, these building blocks are applied to single and portfolio credit risk. This approach generalizes Duffie \& Gârleanu (2001) and is able to produce realistic default correlation and default clustering. We conclude with a specific model where all key building blocks are computed explicitly.
\end{abstract}

Key Words: Term Structure Models, Quadratic Term Structure Models, Shot-noise processes.

JEL Classification: C15,C12, G13, G33 


\title{
Term Structure Models with Shot Noise Effects
}

\author{
Raquel M. Gaspar ${ }^{1}$ And Thorsten Schmidt ${ }^{2}$
}

July 2007

\begin{abstract}
This work proposes term structure models consisting of two parts: a part which can be represented in exponential quadratic form and a shot noise part. These term structure models allow for explicit expressions of various derivatives. In particular, they are very well suited for portfolio credit risk models and for the pricing of CDOs. The goal of the paper is twofold. First, a number of key building blocks useful in term structure modelling are derived in closed-form. Second, these building blocks are applied to single and portfolio credit risk. This approach generalizes Duffie and Gârleanu (2001) and is able to produce realistic default correlation and default clustering. We conclude with a specific model where all key building blocks are computed explicitly.
\end{abstract}

\section{Introduction}

The aim of this paper is to put forth a new class of term structure models, which generalizes affine and jump-diffusion models and still allows for closed form solutions to prices of numerous derivatives. This class is particularly suited to applications in single and portfolio credit risk.

Using the framework of intensity-based models ${ }^{3}$ the proposed class combines the concept of general quadratic term structure (GQTS) models put forth in Gaspar (2004) with a special type of jump-processes, called shot noise processes. The obtained results show that shot noise processes can be paired with any other, independent class of processes and we choose GQTS for the following reasons: First, the considered factors need not to be Gaussian as, e.g., is the case in Chen, Filipović, and Poor (2004). Second, they include affine term structures as a special case. Third, the proposed framework naturally allows for negative correlation of interest rates and default intensities, which is a rather delicate issue in affine models, as pointed out in Chapters 5.8.2 and 5.8.3 of Lando (2004). Fourth, the quadratic class is the largest polynomial class which can be considered without introducing arbitrage opportunities (see Filipović (2002) or Gaspar (2004)).

On the other side, considering shot noise processes includes jump-diffusion models, like Duffie and Gârleanu (2001), as special cases but allows for greater flexibility. In fact, we show how to add several constant intensity jump processes with arbitrary decay rates to any given quadratic diffusion component. Moreover, shot noise patterns are often evident from market data (see Figure 1 for typical patterns of credit default swap (CDS) spreads and Figure 2 for a simulated path of a model of the proposed class). As argued in Mortensen (2006), common jumps in intensities are an efficient way to reproduce observed correlation smiles in the market. In particular, our setup allows to overcome a difficulty in Duffie and Gârleanu

\footnotetext{
${ }^{1}$ Advance Research Center, ISEG, Technical University of Lisbon, Rua Miguel Lupi 20, 1249-078 Lisboa, PORTUGAL. Email: Rmgaspar@iseg.utl.pt

${ }^{2}$ Augustusplatz 10/11, 04109 Leipzig, GERMANY. Email: thorsten.schmidt@math.uni-leipzig.de

${ }^{3}$ See e.g. the survey Schmidt and Stute (2004) or one of the books Lando (2004), Bielecki and Rutkowski (2002), McNeil, Frey, and Embrechts (2005) and Schönbucher (2003).
} 

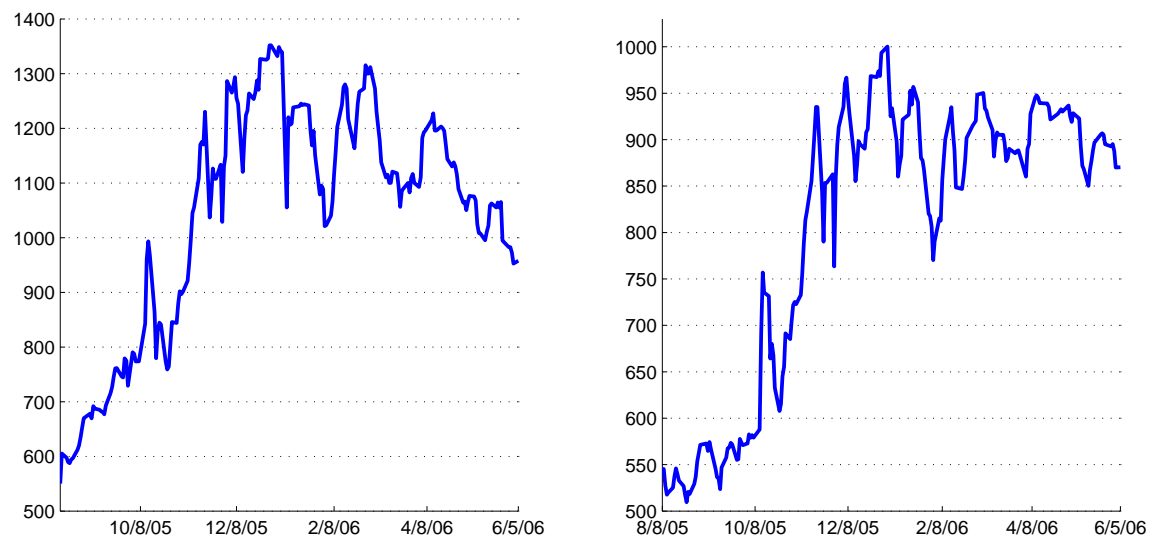

Figure 1: 5-year credit spreads of General Motors (left) and Ford (right). The quoted spread is in basis points and is shown from August 8th, 2005 to June 5th, 2006. Datasource: Bloomberg.

(2001), as in the proposed class the mean-reversion speed of the diffusive and the jump part can be adjusted separately. Finally, for the application to portfolio credit risk the shot noise component allows to obtain a suitable dynamic dependence structure and produces clustered defaults. Needless to say, capturing dynamic dependencies is one of the most important points for modeling portfolio credit derivatives as collateralized debt obligations (CDOs) or First-to-default swaps. In addition, a subclass of the proposed model allows for separate calibration to single name and portfolio credit risk instruments, a useful property in the pricing of portfolio products, see Proposition 4.3. Phrased in market language, the marginal default distribution can be fixed first and the correlation between defaults can then be independently adjusted. For other applications of shot noise processes in finance see, e.g., Altmann, Schmidt, and Stute (2007) and Dassios and Jang (2003).

A recent branch of affine models allows for direct contagion modelling, i.e. a default of one firm has an immediate impact on the default intensity of the other firms. Pioneered by Davis and Lo (2001), there are a number of approaches considering on this topic. We refer to Collin-Dufresne, Goldstein, and Hugonnier (2004), Frey, Prosdocimi, and Runggaldier (2007) and Frey and Runggaldier (2006). The model proposed in this paper excludes this kind of direct contagion. Nonetheless, contagion effects are captured via the shot-noise part as explained above. Being technically simpler, and still capturing typical default correlations, the chosen approach seems to be very suitable for practical applications.

The main goals of this paper are:

- To use GQTS and shot noise processes to model default risk.

- To get explicit solutions (up to ODE systems solving) for all crucial expectations, here called the credit risk building blocks.

- To use these building blocks to obtain key ingredients in credit risk, such as probabilities of default and prices of risk-free and defaultable bonds, CDSs, etc.

- Finally, to propose a multi-firm setup which produces reasonable clustering of defaults and realistic default correlation. 
The paper is organized as follows: in Section 2 we briefly review the GQTS. In Section 3 we present the model and the key building blocks are derived. Section 4 shows the use of the key building blocks providing several applications to the pricing of credit risk. In Section 5 we consider a three-factor model and provide all necessary expressions explicitly.

\section{Risk-free bond market}

For the risk-free bond market we use the GQTS setup studied in Gaspar (2004). This setup assumes as given factors described by a $\mathbb{R}^{m}$-valued stochastic process $\left(Z_{t}\right)_{t \geq 0}$ whose dynamics, under the risk-neutral martingale measure $\mathbb{Q}$, are of a special form. Furthermore, it assumes that the risk-free rate of interest $r$ is quadratic on those factors.

Assumption 2.1. $W$ is an $n$-dimensional standard Brownian motion and $Z$ is the unique strong solution of

$$
d Z_{t}=\alpha\left(t, Z_{t}\right) d t+\sigma\left(t, Z_{t}\right) d W_{t}
$$

Here $\alpha: \mathbb{R}_{+} \times \mathbb{R}^{m} \mapsto \mathbb{R}^{m}$ and $\sigma: \mathbb{R}_{+} \times \mathbb{R}^{m} \mapsto \mathbb{R}^{n \times n}$ are such that

$$
\begin{aligned}
\alpha(t, z) & =d(t)+E(t) z \\
\sigma(t, z) \sigma^{\top}(t, z) & =k_{0}(t)+\sum_{i=1}^{m} k_{i}(t) z_{i}+\sum_{i, j=1}^{m} z_{i} g_{i j}(t) z_{j}
\end{aligned}
$$

with smooth functions $d: \mathbb{R}_{+} \mapsto \mathbb{R}^{m}, E, k_{0}, k_{i}$ and $g_{i j}, i, j=1, \cdots, m$ map $\mathbb{R}_{+}$to $\mathbb{R}^{m \times m}$. Moreover, the risk-free short rate $\left(r_{t}\right)_{t \geq 0}$ is given by

$$
r\left(t, Z_{t}\right)=Z_{t}^{\top} Q(t) Z_{t}+g^{\top}(t) Z_{t}+f(t)
$$

$Q, g$ and $f$ are smooth, mapping $\mathbb{R}_{+}$to $\mathbb{R}^{m \times m}, \mathbb{R}^{m}$ and $\mathbb{R} . Q(t)$ is symmetric ${ }^{4}$ for all $t$.

We classify factors based on their impact on the drift, volatility or the short rate.

\section{Definition 2.2. (Classification of risk-free factors)}

- $Z_{i}$ is a risk-free quadratic-factor if it satisfies at least one of the following requirements:

(i) it has a quadratic impact on the short rate, i.e., there exists $t$ such that $Q_{i}(t) \neq 0$;

(ii) it has a quadratic impact on $\sigma \sigma^{\top}$, i.e., there exist $j$ and $t$ such that $g_{i j}(t) \neq 0$;

(iii) it affects the drift term of the factors satisfying (i) or (ii), i.e., for some $Z_{j}$ satisfying (i) or (ii) we have $E_{j i}(t) \neq 0$, at least for some $t$.

- $Z_{i}$ is a risk-free linear-factor, if it does not satisfy any of (i)-(iii).

We write symbolically $Z_{i} \in Z^{(q)}$, if $Z_{i}$ is a risk-free quadratic factor and $Z_{i} \in Z^{(l)}$ otherwise.

Finally, based upon on the factor's classification, it is possible to derive under what conditions we will have a quadratic term structure of (zero-coupon) risk-free bond prices. To explicitly get the term structure we will always have to solve what we will here define as basic ODE system.

\footnotetext{
${ }^{4}$ The symmetry assumption is not restrictive. Any non-symmetric quadratic form can be rewritten in an equivalent symmetric way with the advantage that the symmetric representation is unique.
} 
Definition 2.3. (Basic ODE System) Denote $\mathcal{T}:=\left\{(t, T) \in \mathbb{R}^{2}: 0 \leq t \leq T\right\}$ and consider functions $A, B$ and $C$ on $\mathcal{T}$ with values in $\mathbb{R}, \mathbb{R}^{m}$ and $\mathbb{R}^{m \times m}$, respectively. For functions $\phi_{1}$ and $\phi_{2}, \phi_{3}$ on $\mathbb{R}^{+}$with values in $\mathbb{R}, \mathbb{R}^{m}$ and $\mathbb{R}^{m \times m}$, respectively, we say that $\left(A, B, C, \phi_{1}, \phi_{2}, \phi_{3}\right)$ solves the basic ODE system if

$$
\begin{aligned}
\frac{\partial A}{\partial t}+d^{\top}(t) B+\frac{1}{2} B^{\top} k_{0}(t) B+\operatorname{tr}\left\{C k_{0}(t)\right\} & =\phi_{1}(t) \\
\frac{\partial B}{\partial t}+E^{\top}(t) B+2 C d(t)+\frac{1}{2} \tilde{B}^{\top} K(t) B+2 C k_{0}(t) B & =\phi_{2}(t) \\
\frac{\partial C}{\partial t}+C E(t)+E^{\top}(t) C+2 C k_{0}(t) C+\frac{1}{2} \tilde{B}^{\top} G(t) \tilde{B} & =\phi_{3}(t)
\end{aligned}
$$

subject to the boundary conditions $A(T, T)=0, B(T, T)=0, C(T, T)=0$. $A, B$ and $C$ should always be evaluated at $(t, T) . E, d, k_{0}$, are the functions from the above definitions (recall (1)-(2)) while

$$
\tilde{B}:=\left(\begin{array}{cccc}
B & 0 & \cdots & 0 \\
0 & B & \cdots & 0 \\
\vdots & & \ddots & \\
0 & \cdots & 0 & B
\end{array}\right), \quad K(t)=\left(\begin{array}{c}
k_{1}(t) \\
\vdots \\
k_{m}(t)
\end{array}\right), \quad G(t)=\left(\begin{array}{ccc}
g_{11}(t) & \cdots & g_{1 m}(t) \\
\vdots & \ddots & \vdots \\
g_{m 1}(t) & \cdots & g_{m m}(t)
\end{array}\right)
$$

where we have $\tilde{B}, K(t) \in \mathbb{R}^{m^{2} \times m}$ and $G(t) \in \mathbb{R}^{m^{2} \times m^{2}}$.

The main result on risk-free bond prices' term structure is the following. ${ }^{5}$

Result 2.4. (Gaspar) Suppose that Assumption 2.1 holds and factors in $Z$ have been reordered as $Z=\left(Z^{(q)}, Z^{(l)}\right)^{\top}$. If for $k_{i}$ and $g_{i j}$ in (2) we have that

$$
k_{i}=\left(\begin{array}{cc}
0 & 0 \\
0 & k_{i}^{(l l)}
\end{array}\right) \forall i \quad \text { and } \quad g_{i j}=\left(\begin{array}{cc}
0 & 0 \\
0 & g_{i j}^{(l l)}
\end{array}\right) \quad \forall i, j \text { s.t. } Z_{i}, Z_{j} \in Z^{(q)},
$$

then the term structure of risk-free zero-coupon bond prices is given by

$$
p(t, T)=\exp \left[A(t, T)+B^{\top}(t, T) Z_{t}+Z_{t}^{\top} C(t, T) Z_{t}\right]
$$

where $(A, B, C, f, g, Q)$ solves the basic ODE system from Definition 2.3. Recall that $f, g$ and $Q$ were given in Equation (3). Moreover, $C$ has only quadratic factors.

\section{Defaultable bond market}

In this section we present the defaultable bond market. To this, we use the well-known framework of doubly stochastic random times. For an introduction to this topic we refer to McNeil, Frey, and Embrechts (2005) or Bielecki and Rutkowski (2002).

\footnotetext{
${ }^{5}$ For the proof and further details we refer to Gaspar (2004)
} 


\subsection{Default events}

The default intensity is modelled as a linear combination of GQTS and shot noise processes. Throughout we assume Assumption 2.1 is in force ${ }^{6}$.

\section{Assumption 3.1. (Default Events and Filtrations)}

Consider a standard Poisson process $\tilde{N}$ with intensity $l^{7}$. Denote the jumping times of $\tilde{N}$ by $\tilde{\tau}_{1}, \tilde{\tau}_{2}, \ldots$ The processes $\eta, J$ and $\mu$ are strictly positive and follow

$$
\begin{aligned}
\eta_{t} & =Z_{t}^{\top} \mathrm{Q}(t) Z_{t}+\mathrm{g}^{\top}(t) Z_{t}+\mathrm{f}(t) \\
J_{t} & =\sum_{\tilde{\tau}_{i} \leq t} Y_{i} h\left(t-\tilde{\tau}_{i}\right),
\end{aligned}
$$

$\mu_{t}=\eta_{t}+J_{t}$. Here $\mathrm{Q}, \mathrm{g}$ and $\mathrm{f}$ are smooth functions mapping $\mathbb{R}_{+}$to $\mathbb{R}^{m \times m}, \mathbb{R}^{m}$ and $\mathbb{R}$, respectively. $\mathrm{Q}(t)$ is symmetric for all $t . Y_{i}, i=1,2, \ldots$ are i.i.d., independent of $W$ and $\tilde{N}$ and $h$ is a differentiable function on $\mathbb{R}^{+}$. The default time $\tau$ is a doubly stochastic random time with intensity $\left(\mu_{t}\right)_{t \geq 0}$.

$J$ is called a shot noise process.

Throughout, denote by $\mathcal{F}^{X}$ the natural filtration generated by a generic process $X$. We classify the market information according to the following filtrations: $\mathcal{F}^{W}$ the information about the diffusion factors; $\mathcal{F}^{J}$ the information about the jump factors; the filtration $\mathcal{H}_{t}:=\sigma\left(\mathbf{1}_{\{\tau>s\}}: 0 \leq s \leq t\right)$, information on the default state; $\mathcal{F}_{t}:=\mathcal{F}_{t}^{W} \vee \mathcal{F}_{t}^{J}=$ $\sigma\left(Z_{s}, J_{s}: 0 \leq s \leq t\right)$, information about all market factors and $\mathcal{G}_{t}:=\mathcal{F}_{t} \vee \mathcal{H}_{t}$, the total information. For future convenience, we also define

$$
\begin{aligned}
H(x) & :=\int_{0}^{x} h(u) d u, \quad D(\theta, x):=\int_{0}^{1} \varphi[\theta H(x(1-u))] d u \\
J(t, T) & :=\sum_{\tilde{\tau}_{i} \leq t} Y_{i} h\left(T-\tilde{\tau}_{i}\right), \quad \tilde{J}(t, T)=\sum_{\tilde{\tau}_{i} \leq t} Y_{i} H\left(T-\tilde{\tau}_{i}\right),
\end{aligned}
$$

where $\varphi(\theta):=\mathbb{E}\left(\exp \left(\theta Y_{1}\right)\right)$. Note that $J(t, t)=J_{t}$ and we set $\tilde{J}_{t}:=\tilde{J}(t, t)$.

Intuitively, the modeling of a quadratic component and a shot noise component leads to the intensity being driven by a predictable component (the quadratic part) as well as by an unpredictable component (the jump part). We note that both $\eta$ and $J$ are assumed to be strictly positive. This assumption is needed because $\mu$ is supposed to be an intensity. This point distinguishes classical interest rate models from reduced-form credit risk models in that in contrast to interest rates, negative intensities show a model inconsistency. Unfortunately, this is the case for some affine intensity models. Even if, through parameter restrictions, positiveness can be guaranteed, this usually comes at the cost of a loss in flexibility. In Proposition 3.3 we show that in our setting positivity can be guaranteed under very mild assumptions. This is a crucial point in favor of quadratic processes over affine processes for modelling the predictable part of the intensity.

For the shot noise part, Figure 2 shows a possible realization of $J$ under $h(x)=\exp (-b x)$. Note that for realistic modeling the decay factor $b$ of the shot noise part is by far larger than the mean reversion speed $\kappa$, which is a key improvement over affine jump-diffusions, as used for example in Duffie and Gârleanu (2001).

2.2 , we give an intensity classification of factors.

\footnotetext{
${ }^{6}$ Taking the same factors $Z$ as for the risk-free process is no loss of generality.

${ }^{7}$ We assume that $W$ and $\tilde{N}$ are adapted to a common filtration and hence are necessarily independent.
} 

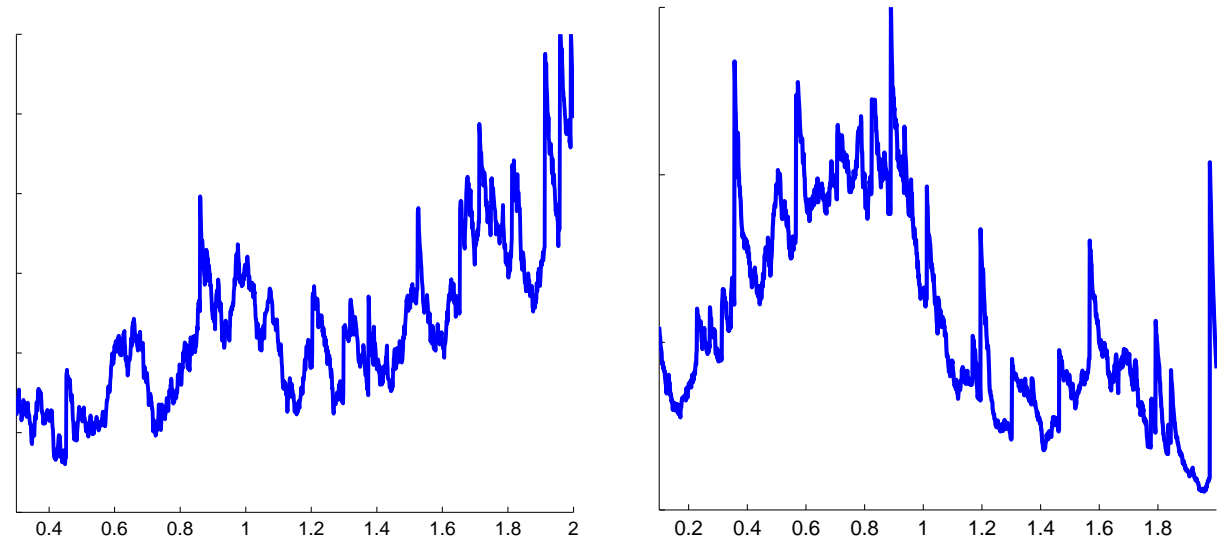

Figure 2: Simulation of $J$ with $h(x)=e^{-b x}$ for $b=30$ (right) and $b=50$ (left), $\chi_{2^{-}}^{2}$ distributed $Y_{i}$ with additional quadratic part. The quadratic part is CIR with with mean reversion speed $\kappa=0.5$, mean reversion level $\theta=1$ and volatility $\sigma=2$.

Definition 3.2. (Classification of intensity factors). We call $Z_{i}$ an intensity quadraticfactor if it satisfies (ii) or (iii) in Definition 2.2 or it has a quadratic impact on $\eta$, i.e. $\exists t \geq 0$ such that $\mathrm{Q}_{i}(t) \neq 0$; We call $Z_{i}$ an intensity linear-factor if it does not satisfy either of these. We write $Z_{i} \in Z_{\eta}^{(q)}, Z_{\eta}^{(l)}$ for the quadratic and linear intensity factors, respectively ${ }^{8}$.

The next proposition gives a condition guaranteeing non-negativity of the default intensity.

Proposition 3.3. Assume that for all $t \geq 0, \mathrm{Q}^{(q q)}(t)$ is symmetric and nonnegative definite, $\mathrm{g}^{(q)}(t)$ lies in the subspace spanned by the columns of $\mathrm{Q}^{(q q)}(t)$ and $\mathrm{f}(t) \geq 0$. Denote by $Z_{\eta *}^{(q)}(t)$ the solution of $\mathrm{Q}^{(q q)}(t) Z_{\eta}^{(q)}(t)=-\frac{1}{2} \mathrm{~g}^{(q)}(t)$. Then $\eta$ is non-negative, if the following holds ${ }^{9}$ :

(i) for $Z_{i} \in Z_{\eta}^{(l)}$, either $Z_{i} \geq 0$ and $\mathrm{g}_{i}^{(l)} \geq 0$ or $Z_{i} \leq 0$ and $\mathrm{g}_{i}^{(l)} \leq 0$,

(ii) for all $t \geq 0, \frac{1}{2} g^{(q) \top} Z_{\eta^{*}}^{(q)}(t)+\mathrm{f}(t) \geq 0$.

Proof. Let $Z_{i}$ be a linear factor, say $Z_{i} \in Z_{\eta}^{(l)}$. As $\mathrm{f} \geq 0$, the result follows trivially from the fact that, by Definition 3.2, $\mathrm{Q}^{(l l)}(t)=0$ and $\mathrm{Q}^{(l q)}(t)=\left(\mathrm{Q}^{(q l)}(t)\right)^{\top}=0$ for all $t$. For a quadratic factor we need to study $\mathrm{Q}^{(q q)}$ and $\mathrm{g}^{(q)}$. Since $Q^{(q q)}$ is nonnegative definite, $Z_{*}^{(q)}$ is the minimum of the polynomial $Z_{\eta}^{(q) \top} \mathrm{Q}^{(q q)} Z_{\eta}^{(q)}+\mathrm{g}^{(q) \top} Z_{\eta}^{(q)}$, and hence $\frac{1}{2} \mathrm{~g}^{(q) \top} Z_{\eta *}^{(q)}+\mathrm{f} \geq 0$ guarantees nonnegativity.

Typically, shot-noise processes are not Markovian. Still, from a computational point of view Markovianity could be preferable. We provide a clear classification.

Proposition 3.4. Assume that for all $x \in[0, \infty) h(x) \neq 0$. Then the process $\left(J_{t}\right)_{t \geq 0}$ is Markovian, if and only if $h$ is of the form $h(t)=a e^{-b t}$. In this case $(J, \eta)$ is Markovian.

Proof. It is clear that for $b=0$ the process is Markovian, so we need to consider the case where $h$ is not constant. Assume w.l.o.g. that $h(0)=1$. To show that $J$ is Markovian we compute the conditional expectation. Consider $s<t$ and recall $\mathcal{F}_{t}^{J}:=\sigma\left\{J_{s}: s \leq t\right\}$. Then

\footnotetext{
${ }^{8}$ We use the symbolic notation $\bar{Z}^{(q)}=Z^{(q)} \cup Z_{\eta}^{(q)}$ and $\bar{Z}^{(l)}=Z^{(l)} \cap Z_{\eta}^{(l)}$, whenever the factors must be ordered according to both their impact on $r$ and on $\eta$.

${ }^{9}$ Here we use the following symbolic notation for $\mathrm{g}$ and $\mathrm{Q}: \quad \mathrm{g}=\left(\begin{array}{l}\mathrm{g}^{(q)} \\ \mathrm{g}^{(l)}\end{array}\right), \mathrm{Q}=\left(\begin{array}{ll}\mathrm{Q}^{(q q)} & \mathrm{Q}^{(q l)} \\ \mathbf{Q}^{(l q)} & \mathrm{Q}^{(l l)}\end{array}\right)$.
} 


$$
\mathbb{E}^{\mathbb{Q}}\left[J_{t} \mid \mathcal{F}_{s}^{J}\right]=\sum_{i=1}^{\tilde{N}_{s}} Y_{i} h\left(t-\tilde{\tau}_{i}\right)+\mathbb{E}^{\mathbb{Q}}\left[\sum_{i=\tilde{N}_{s}+1}^{\tilde{N}_{t}-\tilde{N}_{s}+\tilde{N}_{s}} Y_{i} h\left(t-\tilde{\tau}_{i}\right) \mid \mathcal{F}_{s}^{J}\right] .
$$

As $\tilde{N}$ has independent increments and the $Y_{i}$ are identically distributed, we obtain

$$
\mathbb{E}^{\mathbb{Q}}\left[\sum_{i=j+1}^{\tilde{N}_{t}-\tilde{N}_{s}+j} Y_{i} h\left(t-\tilde{\tau}_{i}\right) \mid \tilde{N}_{s}=j\right]=\mathbb{E}^{\mathbb{Q}}\left[\sum_{i=1}^{\tilde{N}_{t-s}} Y_{i} h\left(t-\tilde{\tau}_{i}\right)\right]=: f(s, t) .
$$

Hence $(9)=\sum_{i=1}^{\tilde{N}_{s}} Y_{i} h\left(t-\tilde{\tau}_{i}\right)+f(s, t)$. As $f(s, t)$ is deterministic, necessary for Markovianity is that there exists a (measurable) function $F(t, s, x)$, such that

$$
\sum_{i=1}^{\tilde{N}_{s}} Y_{i} h\left(t-\tilde{\tau}_{i}\right)=F\left(t, s, J_{s}\right)=F\left(t, s, \sum_{i=1}^{\tilde{N}_{s}} Y_{i} h\left(s-\tilde{\tau}_{i}\right)\right) .
$$

Note that each $Y_{i}$ is independent of all the other appearing terms. We will exploit this property to analyze the behavior of $F$. Fix $j$ and consider (10) on the set $\left\{\tilde{N}_{t}>j\right\}$. Taking the conditional expectation of (10) w.r.t. $Y_{j}=y$ gives

$$
\mathbb{E}^{\mathbb{Q}}\left(y h\left(t-\tilde{\tau}_{j}\right)+\sum_{i=1, i \neq j}^{\tilde{N}_{s}} Y_{i} h\left(t-\tilde{\tau}_{i}\right)\right)=\mathbb{E}^{\mathbb{Q}}\left(F\left(t, s, y h\left(s-\tilde{\tau}_{j}\right)+\sum_{i=1, i \neq j}^{\tilde{N}_{s}} Y_{i} h\left(s-\tilde{\tau}_{i}\right)\right)\right) .
$$

Deriving w.r.t. $y$ shows that

$$
\mathbb{E}^{\mathbb{Q}}\left(h\left(t-\tilde{\tau}_{j}\right)\right)=\mathbb{E}^{\mathbb{Q}}\left[F_{x}\left(t, s, y h\left(s-\tilde{\tau}_{j}\right)+\sum_{i=1, i \neq j}^{\tilde{N}_{s}} Y_{i} h\left(s-\tilde{\tau}_{i}\right)\right) h\left(s-\tilde{\tau}_{j}\right)\right],
$$

where we denoted the partial derivative of $F$ w.r.t. $x$ by $F_{x}$. As the l.h.s. does not depend on $y, F_{x}(t, s, x)$ must be constant in $x$, and thus $F$ must be of the form $\alpha(t, s)+\beta(t, s) x$. Examining $F$ on the set $\left\{\tilde{N}_{t}=0\right\}$, we see that $\alpha(t, s)$ must necessarily be 0 . In the next step we determine $\beta$. From Equation (10) we obtain, for any $i, h\left(t-\tilde{\tau}_{i}\right)=\beta(s, t) h\left(s-\tilde{\tau}_{i}\right)$. Hence, $\beta(s, t)=h(t-y) / h(s-y)$ for any $y \geq 0$, and so $b(s, t)=h(t) / h(s)$. From this we have $h(t-y) / h(s-y)=h(t) / h(s)$, for all $t, s, y \geq 0$. By letting $s=y$ we obtain that $h(t-y)=h(0) h(t) / h(y)$ and so $h(t+y)=h(t) h(y) / h(0)$. We conclude $h^{\prime}(y)=$ $h^{\prime}(0) h(y) / h(0)$. Therefore $h$ is of the form $a e^{-b y}$.

For the converse, note that for $h(y)=e^{-b y}, \sum_{i=1}^{\tilde{N}_{t}} Y_{i} h\left(t-\tilde{\tau}_{i}\right)=h(t) \sum_{i=1}^{\tilde{N}_{t}} Y_{i} h\left(-\tilde{\tau}_{i}\right)$, and hence $J$ is Markovian. Finally, independence of $J$ and $\eta$ implies Markovianity of $(J, \eta)$.

It is clear that Markovianity of $\mu$ itself only holds in very special cases. One well-known case is, when $\eta$ is affine, has mean reversion speed $b$ and $h(t)=\exp (-b t)$.

Remark 3.5. In the Markovian case the terms in (7)-(8) simplify considerably. For $h(x)=$ $e^{-b x}$ we have that $H(x)=\frac{1}{b}\left(1-e^{-b x}\right)$. Thus, $H\left(T-\tilde{\tau}_{i}\right)-H\left(t-\tilde{\tau}_{i}\right)=h\left(t-\tilde{\tau}_{i}\right) H(T-t)$, as well as $\tilde{J}_{t}-\tilde{J}(t, T)=-H(T-t) J_{t}$.

\subsection{Building blocks}

In this section we give analytical expressions, up to the solution of ODE systems, of what we consider key building blocks of credit risk models. We start with an important Lemma.

Lemma 3.6. Consider smooth $G, F: \mathbb{R}_{+} \times \mathbb{R}^{m} \mapsto \mathbb{R}$ where $F(t, z)=\phi_{1}(t)+\phi_{2}^{\top}(t) z+$ $z^{\top} \phi_{3}(t) z$. Then,

$$
\mathbb{E}^{\mathbb{Q}}\left[G\left(Z_{T}, T\right) e^{-\int_{t}^{T} F\left(s, Z_{s}\right) d s} \mid \mathcal{F}_{t}\right]=g\left(t, Z_{t}, T\right) e^{A(t, T)+B^{\top}(t, T) Z_{t}+Z_{t}^{\top} C(t, T) Z_{t}},
$$


where $\left(A, B, C, \phi_{1}, \phi_{2}, \phi_{3}\right)$ solve the basic ODE system of Definition 2.3 and $g$ the PDE

$$
\left\{\begin{aligned}
\frac{\partial g}{\partial t}+\sum_{i} \frac{\partial g}{\partial z_{i}} \alpha_{i}+\frac{1}{2} \sum_{i j}\left(\frac{\partial^{2} g}{\partial z_{i} \partial z_{j}}+\frac{\partial g}{\partial z_{i}} \frac{\partial h}{\partial z_{j}}+\frac{\partial g}{\partial z_{j}} \frac{\partial h}{\partial z_{i}}\right) \sigma_{i} \sigma_{j} & =0 \\
g(T, z, T) & =G(z, T) .
\end{aligned}\right.
$$

Proof. Let $y\left(t, Z_{t}, T\right)=\mathbb{E}^{\mathbb{Q}}\left[G\left(T, Z_{T}, T\right) \exp \left(-\int_{t}^{T} F\left(s, Z_{s}\right) d s\right) \mid \mathcal{F}_{t}\right]$. Then

$$
\left\{\begin{aligned}
\frac{\partial y}{\partial t}+\sum_{i} \frac{\partial y}{\partial z} \alpha_{i}+\frac{1}{2} \sum_{i j} \frac{\partial^{2} y}{\partial z_{i} \partial z_{j}} \sigma_{i} \sigma_{j} & =F y \\
y(T, z, T) & =G(z, T)
\end{aligned}\right.
$$

where all partial derivatives should be evaluated at $(t, T)$ and $\alpha$ and $\sigma$ are the drift and diffusion $Z$ as defined in (2). Note that, if the above expectation is of the form $y(t, z, T)=$ $g(t, z, T) e^{A(t, T)+B^{\top}(t, T) z+z^{\top} C(t, T) z}=g(t, z, T) e^{h(t, z, T)}, z \in \mathbb{R}^{m}$, we have the following partial derivatives

$$
\begin{gathered}
\frac{\partial y}{\partial t}=\frac{\partial g}{\partial t} \cdot e^{h}+\frac{\partial h}{\partial t} \cdot g \cdot e^{h}=\frac{\partial g}{\partial t} \cdot e^{h}+\frac{\partial h}{\partial t} \cdot y, \quad \frac{\partial y}{\partial z_{i}}=\frac{\partial g}{\partial z_{i}} e^{h}+g \cdot \frac{\partial h}{\partial z_{i}} \cdot e^{h}=\frac{\partial g}{\partial z_{i}} e^{h}+\frac{\partial h}{\partial z_{i}} \cdot y \\
\frac{\partial^{2} y}{\partial z_{i} \partial z_{j}}=\left[\frac{\partial^{2} g}{\partial z_{i} \partial z_{j}} \cdot e^{h}+\frac{\partial g}{\partial z_{i}} \frac{\partial h}{\partial z_{j}} \cdot e^{h}+\frac{\partial g}{\partial z_{j}} \frac{\partial h}{\partial z_{i}} e^{h}+g\left(\frac{\partial^{2} h}{\partial z_{i} \partial z_{j}} \cdot e^{h}+\frac{\partial h}{\partial z_{i}} \frac{\partial h}{\partial z_{j}} \cdot e^{h}\right)\right] \\
=\frac{\partial^{2} g}{\partial z_{i} \partial z_{j}} \cdot e^{h}+\frac{\partial g}{\partial z_{i}} \frac{\partial h}{\partial z_{j}} \cdot e^{h}+\frac{\partial g}{\partial z_{j}} \frac{\partial h}{\partial z_{i}} \cdot e^{h}+\frac{\partial^{2} h}{\partial z_{i} \partial z_{j}} \cdot y+\frac{\partial h}{\partial z_{i}} \frac{\partial h}{\partial z_{j}} \cdot y \\
\text { and }(12)=\left\{\begin{array}{r}
\frac{\partial g}{\partial t} \cdot e^{h}+\frac{\partial h}{\partial t} \cdot y+\sum_{i}\left(\frac{\partial g}{\partial z_{i}} e^{h}+\frac{\partial h}{\partial z_{i}} \cdot y\right) \alpha_{i}+ \\
\frac{1}{2} \sum_{i j}\left(\frac{\partial^{2} g}{\partial z_{i} \partial z_{j}} \cdot e^{h}+\frac{\partial g}{\partial z_{i}} \frac{\partial h}{\partial z_{j}} \cdot e^{h}+\frac{\partial g}{\partial z_{j}} \frac{\partial h}{\partial z_{i}} \cdot e^{h}\right) \sigma_{i} \sigma_{j} \\
+\frac{1}{2} \sum_{i j}\left(\frac{\partial^{2} h}{\partial z_{i} \partial z_{j}} \cdot y+\frac{\partial h}{\partial z_{i}} \frac{\partial h}{\partial z_{j}} \cdot y\right) \sigma_{i} \sigma_{j}=F y \\
y(T, z, T)=G(z, T) .
\end{array}\right.
\end{gathered}
$$

By separation of variables (in $e^{h}$ and $h$ terms) the above PDE is equivalent to the system

$$
\begin{aligned}
& \left\{\begin{array}{r}
\frac{\partial h}{\partial t}+\sum_{i} \frac{\partial h}{\partial z_{i}} \alpha_{i}+\frac{1}{2} \sum_{i j}\left(\frac{\partial^{2} h}{\partial z_{i} \partial z_{j}}+\frac{\partial h}{\partial z_{i}} \frac{\partial h}{\partial z_{j}}\right) \sigma_{i} \sigma_{j}=F \\
h(T, z, T)=0
\end{array}\right. \\
& \left\{\begin{aligned}
\frac{\partial g}{\partial t}+\sum_{i} \frac{\partial g}{\partial z_{i}} \alpha_{i}+\frac{1}{2} \sum_{i j}\left(\frac{\partial^{2} g}{\partial z_{i} \partial z_{j}}+\frac{\partial g}{\partial z_{i}} \frac{\partial h}{\partial z_{j}}+\frac{\partial g}{\partial z_{j}} \frac{\partial h}{\partial z_{i}}\right) \sigma_{i} \sigma_{j} & =0 \\
g(T, z, T) & =G(z, T)
\end{aligned}\right.
\end{aligned}
$$

To prove the result it remains to show that $h(t, z, T)=A(t, T)+B^{\top}(t, T) z+z^{\top}(t, T) z$, with $A, B$ and $C$ from the basic ODE system of Definition 2.3, solves the PDE (13). This follows from $\frac{\partial h}{\partial t}=\frac{\partial \bar{A}}{\partial t}+\frac{\partial \bar{B}^{\top}}{\partial t} z+z^{\top} \frac{\partial \bar{C}}{\partial t} z, \frac{\partial h}{\partial z_{i}}=\left(\bar{B}_{i}+2 \bar{C}_{i} z\right), \frac{\partial^{2} h}{\partial z_{i} \partial z_{j}}=2 \bar{C}_{i j}$ and the fact that the PDE (13) becomes a separable equation equivalent to the basic ODE system.

We introduce the notion of an interlinked ODE system.

Definition 3.7. (Interlinked ODE system) Consider smooth functions $a, b, c, B, C$ on $\mathcal{T}$ with values in $\mathbb{R}, \mathbb{R}^{m}, \mathbb{R}^{m \times m}, \mathbb{R}^{m}$ and $\mathbb{R}^{m \times m}$, and smooth functions $\phi_{1}, \phi_{2}$ and $\phi_{3}$ on $\mathbb{R}_{+}$ with values in $\mathbb{R}, \mathbb{R}^{m}$ and $\mathbb{R}^{m \times m}$ respectively. We say that $\left(a, b, c, B, C, \phi_{1}, \phi_{2}, \phi_{3}\right)$ solves the interlinked ODE system if it solves

$$
\begin{aligned}
\frac{\partial a}{\partial t}+d^{\top}(t) b+B^{\top} k_{0}(t) b+\operatorname{tr}\left\{c k_{0}(t)\right\} & =0 \\
\frac{\partial b}{\partial t}+E^{\top}(t) b+2 c d(t)+\frac{1}{2} \tilde{B}^{\top} k_{0}(t) b+2 c k_{0}(t) B+2 C k_{0}(t) b & =0 \\
\frac{\partial c}{\partial t}+c E(t)+E^{\top}(t) c+4 C k_{0}(t) c+\frac{1}{2} \tilde{B}^{\top} G(t) \tilde{b} & =0
\end{aligned}
$$


subject to the boundary conditions $a(T, T)=\phi_{1}(T), b(T, T)=\phi_{2}(T), c(T, T)=\phi_{3}(T)$. $a, b, c$ and $B, C$ should always be evaluated at $(t, T) . E, d, k_{0}$, are the functions from (2) while $\tilde{B}, K \in \mathbb{R}^{m^{2} \times m}$ and $G \in \mathbb{R}^{m^{2} \times m^{2}}$ are as in (4).

The following theorem gives the building blocks for pricing credit derivatives in our setup.

Theorem 3.8. Let $x=T-t$ and consider $r$ as in (3), $J$ as in (6), $\eta$ as in (5) and $\theta \in \mathbb{R}$. For (ii) we also require existence of $D(\theta, x)$ and for $(v)$ that $D$ is bounded in some neighborhood of $x$. Then,

$$
\begin{aligned}
S_{\eta}(\theta, t, T) & :=\mathbb{E}^{\mathbb{Q}}\left[e^{-\int_{t}^{T} \theta \eta_{s} d s} \mid \mathcal{F}_{t}^{W}\right] \\
& =\exp \left(\mathcal{A}(\theta, t, T)+\mathcal{B}^{\top}(\theta, t, T) Z_{t}+Z_{t}^{\top} \mathcal{C}(\theta, t, T) Z_{t}\right) \\
S_{J}(\theta, t, T) & :=\mathbb{E}^{\mathbb{Q}}\left[e^{-\int_{t}^{T} \theta J_{s} d s} \mid \mathcal{F}_{t}^{J}\right]=\exp \left(\theta\left(\tilde{J}_{t}-\tilde{J}(t, T)\right)+l x[D(\theta, x)-1]\right) \\
\bar{S}_{\eta}(\theta, t, T) & :=\mathbb{E}^{\mathbb{Q}}\left[e^{-\int_{t}^{T} r_{s}+\theta \eta_{s} d s} \mid \mathcal{F}_{t}^{W}\right] \\
& =\exp \left(\bar{A}(\theta, t, T)+\bar{B}^{\top}(\theta, t, T) Z_{t}+Z^{\top}(t) \bar{C}(\theta, t, T) Z_{t}\right) \\
\Gamma_{\eta}(\theta, t, T) & :=\mathbb{E}^{\mathbb{Q}}\left[\theta \eta_{T} e^{-\int_{t}^{T} \theta \eta_{s} d s} \mid \mathcal{F}_{t}^{W}\right] \\
& =\left(a(\theta, t, T)+b^{\top}(\theta, t, T) Z_{t}+Z_{t}^{\top} c(\theta, t, T) Z_{t}\right) S_{\eta}(\theta, t, T) \\
\Gamma_{J}(\theta, t, T) & :=\mathbb{E}^{\mathbb{Q}}\left[\theta J_{T} e^{-\int_{t}^{T} \theta J_{s} d s} \mid \mathcal{F}_{t}^{J}\right] \\
& =S_{J}(\theta, t, T)\left\{\theta J(t, T)-l \cdot\left[D(\theta, x)(1-x)-1+x \varphi_{Y}(\theta H(x))\right]\right\} \\
\bar{\Gamma}_{\eta}(\theta, t, T) & :=\mathbb{E}^{\mathbb{Q}}\left[\theta \eta_{T} e^{-\int_{t}^{T} r_{s}+\theta \eta_{s} d s} \mid \mathcal{F}_{t}^{W}\right] \\
& =\left(\bar{a}(\theta, t, T)+\bar{b}^{\top}(\theta, t, T) Z_{t}+Z_{t}^{\top} \bar{c}(\theta, t, T) Z_{t}\right) \cdot \bar{S}_{\eta}(\theta, t, T)
\end{aligned}
$$

where $(\mathcal{A}, \mathcal{B}, \mathcal{C}, \theta \mathrm{f}, \theta \mathrm{g}, \theta \mathrm{Q})$ and $(\bar{A}, \bar{B}, \bar{C}, f+\theta \mathrm{f}, g+\theta \mathrm{g}, Q+\theta \mathrm{Q})$ solve the basic ODE system of Definition 2.3, while $(a, b, c, \mathcal{B}, \mathcal{C}, \theta \mathrm{f}, \theta \mathrm{g}, \theta \mathrm{Q})$ and $(\bar{a}, \bar{b}, \bar{c}, \bar{B}, \bar{C}, \theta \mathrm{f}, \theta \mathrm{g}, \theta \mathrm{Q})$ solve the interlinked system of Definition (3.7). Furthermore,

$$
\begin{array}{rll}
\text { (vii) } & S(\theta, t, T) & :=\mathbb{E}^{\mathbb{Q}}\left[e^{-\int_{t}^{T} \theta \mu_{s} d s} \mid \mathcal{F}_{t}\right]=S_{\eta}(\theta, t, T) S_{J}(\theta, t, T) \\
(\text { viii) } & \bar{S}(\theta, t, T) & :=\mathbb{E}^{\mathbb{Q}}\left[e^{-\int_{t}^{T} r_{s}+\theta \mu_{s} d s} \mid \mathcal{F}_{t}\right]=\bar{S}_{\eta}(\theta, t, T) S_{J}(\theta, t, T) \\
(\text { ix) } & \Gamma(\theta, t, T) & :=\mathbb{E}^{\mathbb{Q}}\left[\theta \mu_{T} e^{-\int_{t}^{T} \theta \mu_{s} d s} \mid \mathcal{F}_{t}\right]=\Gamma_{\eta}(\theta, t, T) S_{J}(\theta, t, T)+\Gamma_{J}(\theta, t, T) S_{\eta}(\theta, t, T) \\
\text { (x) } & \bar{\Gamma}(\theta, t, T) & :=\mathbb{E}^{\mathbb{Q}}\left[\theta \mu_{T} e^{-\int_{t}^{T} r_{s}+\theta \mu_{s} d s} \mid \mathcal{F}_{t}\right]=\bar{\Gamma}_{\eta}(\theta, t, T) S_{J}(\theta, t, T)+\Gamma_{J}(\theta, t, T) \bar{S}_{\eta}(\theta, t, T)
\end{array}
$$

For the case $\theta=1$ we define the shorter notation, $S_{\eta}(t, T):=S_{\eta}(1, t, T)$ and similarly for all the other terms.

Proof. Note that (i) follows from (iii) and (iv) follows from (vi), taking $f(t)=0, g(t)=$ $0, Q(t)=0$ for all $t$, i.e. taking $r$ to be identical zero.

(ii). Recall the notation of $\tilde{J}$ from (8). By definition,

$$
S_{J}(\theta, t, T)=e^{\theta\left(\tilde{J}_{t}-\tilde{J}(t, T)\right)} \mathbb{E}^{\mathbb{Q}}\left[\exp \left(-\sum_{\tilde{\tau}_{i} \in(t, T]} \theta Y_{i} \int_{t}^{T} \mathbf{1}_{\left\{\tilde{\tau}_{i} \leq u\right\}} h\left(u-\tilde{\tau}_{i}\right) d u\right) \mid \mathcal{F}_{t}^{J}\right] .
$$


Recall (7) and observe that $\mathbb{E}^{\mathbb{Q}}\left[\exp \left(-Y_{1} \theta H\left(x\left(1-\eta_{1}\right)\right)\right)\right]=\int_{0}^{1} \varphi_{Y}(\theta H(x(1-u))) d u=$ $D(\theta, x)$. The result follows noting that the expectation in (17) computes to

$$
e^{-l x}+\sum_{k=1}^{\infty} e^{-l x} \frac{(l x)^{k}}{k !} \mathbb{E}^{\mathbb{Q}}\left[\exp \left(-\sum_{i=1}^{k} Y_{i} \theta H\left(x\left(1-\eta_{i}\right)\right)\right)\right]=e^{l x(D(\theta, x)-1)} .
$$

(iii). Again, by definition

$$
\begin{aligned}
\bar{S}(\theta, t, T) & =\mathbb{E}^{\mathbb{Q}}\left[e^{-\int_{t}^{T}\left(Z_{s}^{\top}\left(Q+\theta \mathrm{Q}^{c}(s)\right) Z_{s}+\left(g+\theta \mathrm{g}^{c}(s)\right)^{\top} Z_{s}+\left(f+\theta \mathrm{f}^{c}(s)\right) d s\right)} \mid \mathcal{F}_{t}^{W}\right] \\
& =\exp \left\{\bar{A}(\theta, t, T)+\bar{B}^{\top}(\theta, t, T) Z_{t}+Z_{t}^{\top} \bar{C}(\theta, t, T) Z_{t}\right\} .
\end{aligned}
$$

From Result 2.4 it follows that $(\bar{A}, \bar{B}, \bar{C}, f+\theta \mathrm{f}, g+\theta \mathrm{g}, Q+\theta \mathrm{Q})$ solve the basic system of ODEs from Definition 2.3.

$(v)$. Recall the notations for $\tilde{J}(t, T)$ and $J(t, T)$ introduced in (8). Then

$$
\begin{aligned}
& \Gamma_{J}(\theta, t, T)=\mathbb{E}^{\mathbb{Q}}\left[\theta\left(\sum_{\tilde{\tau}_{i} \leq t} Y_{i} h\left(T-\tilde{\tau}_{i}\right)+\sum_{\tilde{\tau}_{i} \in(t, T]} Y_{i} h\left(T-\tilde{\tau}_{i}\right)\right) e^{-\int_{t}^{T} \theta J_{s} d s} \mid \mathcal{F}_{t}^{J}\right] \\
& =\theta J(t, T) S_{J}(\theta, t, T)+e^{\theta\left(\tilde{J}_{t}-\tilde{J}(t, T)\right)} \mathbb{E}^{\mathbb{Q}}\left[\theta \sum_{\tilde{\tau}_{i} \in(t, T]} Y_{i} h\left(T-\tilde{\tau}_{i}\right) e^{-\theta \int_{t}^{T} \sum_{\tilde{\tau}_{i} \in(t, s]} Y_{i} H\left(s-\tilde{\tau}_{i}\right) d s} \mid \mathcal{F}_{t}^{J}\right]
\end{aligned}
$$

Letting $\tilde{J}^{t}(s):=\sum_{\tilde{\tau}_{i} \in(t, s]} Y_{i} h\left(s-\tilde{\tau}_{i}\right)$, we obtain that

$$
\mathbb{E}^{\mathbb{Q}}\left[\sum_{\tilde{\tau}_{i} \in(t, T]} Y_{i} h\left(T-\tilde{\tau}_{i}\right) e^{-\theta \int_{t}^{T} \sum_{\tilde{\tau}_{i} \in(t, s]} Y_{i} H\left(s-\tilde{\tau}_{i}\right) d s} \mid \mathcal{F}_{t}^{J}\right]=\mathbb{E}^{\mathbb{Q}}\left[\tilde{J}^{t}(T) e^{-\theta \int_{t}^{T} \tilde{J}^{t}(s) d s} \mid \mathcal{F}_{t}^{J}\right]
$$

Note that $H$ is continuous and recall (18). As $D(\theta, x)$ is bounded in a neighborhood of $x$, we obtain $\frac{\partial}{\partial x} e^{l x(\tilde{D}(\theta, x)-1)}=\frac{\partial}{\partial x} \mathbb{E}^{\mathbb{Q}}\left(e^{-\int_{t}^{T} \theta \tilde{J}^{t}(s) d s} \mid \mathcal{F}_{t}^{J}\right)=-\mathbb{E}^{\mathbb{Q}}\left(\theta \tilde{J}_{T}^{t} \cdot e^{-\int_{t}^{T} \theta \tilde{J}^{t}(s) d s} \mid \mathcal{F}_{t}^{J}\right)$.

Using (7) we have $\frac{\partial}{\partial x} D(\theta, x)=\int_{0}^{1} \varphi_{Y}^{\prime}(\theta H(x u)) \cdot \theta h(x u) \cdot u d u=\varphi_{Y}(\theta H(x))-D(\theta, x)$. Thus, $\frac{\partial}{\partial x} e^{l x(D(\theta, x)-1)}=e^{l x(D(\theta, x)-1)} \cdot l \cdot\left[D(\theta, x)(1-x)-1+x \varphi_{Y}(\theta H(x))\right]$. Finally noticing that $e^{\left\{\theta\left(\tilde{J}_{t}-\tilde{J}(t, T)\right\}\right.} e^{\{l x(\tilde{D}(\theta, x)-1)\}}=S_{J}(\theta, t, T)$, we conclude.

$(v i)$. Applying Lemma 3.6 with $y(t, T)=\mathbb{E}^{\mathbb{Q}}\left[\theta \eta_{T} e^{-\int_{t}^{T} r_{u}+\theta \eta_{u} d u} \mid \mathcal{F}_{t}^{W}\right]$ and $G(T, z)=$ $\theta \eta(T, z)$ leads to $\mathbb{E}^{\mathbb{Q}}\left[G\left(Z_{T}, T\right) e^{-\int_{t}^{T} r_{s}+\mu_{s} d s} \mid \mathcal{G}_{t}\right]=g\left(t, Z_{t}, T\right) \underbrace{e^{\bar{A}(t, T)+\bar{B}^{\top}(t, T) Z_{t}+Z_{t}^{\top} \bar{C}(t, T) Z_{t}}}_{\bar{S}_{\eta}(\theta, t, T)}$ where $g$ solves $\left\{\begin{aligned} \frac{\partial g}{\partial t}+\sum_{i} \frac{\partial g}{\partial z_{i}} \alpha_{i}+\frac{1}{2} \sum_{i j}\left(\frac{\partial^{2} g}{\partial z_{i} \partial z_{j}}+\frac{\partial g}{\partial z_{i}} \frac{\partial h}{\partial z_{j}}+\frac{\partial g}{\partial z_{j}} \frac{\partial h}{\partial z_{i}}\right) \sigma_{i} \sigma_{j} & =0 \\ g(T, z, T) & =\eta(T, z) .\end{aligned}\right.$

It remains to show that $g(t, z, T)=\bar{a}(\theta, t, T)+\bar{b}^{\top}(\theta, t, T) z+z^{\top} \bar{c}(\theta, t, T) z$ solves the interlinked ODE system with $(\bar{a}, \bar{b}, \bar{c}, \bar{B}, \bar{C}, f+\theta \mathrm{f}, f+\theta \mathrm{f}, f+\theta \mathrm{f})$. To see this, simply compute $\frac{\partial g}{\partial t}=\frac{\partial \bar{a}}{\partial t}+\frac{\partial \bar{b}}{\partial t} z+z^{\top} \frac{\partial \bar{c}}{\partial t} z, \frac{\partial g}{\partial z_{i}}=\bar{b}_{i}+2 \bar{c}_{i} z, \frac{\partial^{2} g}{\partial z_{i} \partial z_{j}}=2 \bar{c}_{i j}$.

Then, replacing all these partial derivatives and using equations (5) and (2) for $\eta, \alpha$ and $\sigma \sigma^{\top}$, we get an equivalent $\mathrm{PDE}$, which in vector notation becomes ${ }^{10}$

$$
\left\{\begin{array}{r}
\frac{\partial \bar{a}}{\partial t}+\frac{\partial \bar{b}}{\partial t} z+z^{\top} \frac{\partial \bar{c}}{\partial t} z+d^{\top} \bar{b}+\left(E^{*} \bar{b}\right) z+(2 \bar{c} d) z+\frac{1}{2}\left[\bar{B}^{\top} k_{0} b+2 \operatorname{tr}\left\{\bar{c} K_{0}\right\}+(\tilde{\bar{B}} \top K \bar{b}) z\right] \\
+z^{\top}(\bar{c} E) z+z^{\top}\left(E^{*} \bar{c}\right) z+\frac{1}{2}\left[\left(2 \bar{C} k_{0} \bar{b}+2 \bar{c} k_{0} \bar{B}\right) z+z^{\top}\left(4 \bar{C} k_{0} \bar{c}\right) z+z^{\top}(\tilde{\bar{B}} G \tilde{\bar{b}}) z\right]=0 \\
g(T, z, T)=\theta \eta(T, z)
\end{array}\right.
$$

${ }^{10}$ Terms of order higher than two are omitted from the equation since the final solution must set those terms equal to zero and they are hard to write in vector notation. 
This PDE is separable into terms independent of $z$, linear in $z$ and quadratic in $z$ and is equivalent to the interlinked ODE system of Definition 3.7. For the boundary conditions, we note $g(T, z, T)=\theta \eta(T, z)$, thus $\bar{a}(\theta, T, T)+\bar{b}^{\top}(\theta, T, T) z+z^{\top} \bar{c}(\theta, T, T) z=z^{\top} \mathrm{Q}(T) z+$ $\mathrm{g}^{\top}(T) z+\mathrm{f}(T)$ and this implies $\bar{a}(\theta, T, T)=\theta \mathrm{f}(T), \bar{b}(\theta, T, T)=\theta \mathrm{g}(T)$ and $\bar{c}(\theta, T, T)=\theta \mathrm{Q}(t)$. we conclude with $\bar{\Gamma}(\theta, t, T)=\left(\bar{a}(\theta, t, T)+\bar{b}^{\top}(\theta, t, T) z+z^{\top} \bar{c}(\theta, t, T) z\right) \cdot \bar{S}_{\eta}(\theta, t, T)$.

$(v i i)-(x)$ follow from $(i)-(v i)$ by independence of $W$ and $\mathcal{F}^{J}$ and with $\mu=\eta+J$.

The up to now computed expressions were of general interest and may be applied to any term structure. For example, it is now straightforward to compute bond prices in a (riskfree) quadratic shot noise term structure model. However, the next section applies Theorem 3.8 to credit risk, which has been the paper's main motivation.

\section{Applications to the pricing of credit risk}

\subsection{Single name issues}

Based on the building blocks of the previous section we are able to obtain a number of formulas relevant for pricing credit risk:

Survival probabilities. The survival probabilities are the key ingredient for pricing several credit risky securities. On $\{\tau>t\}$, they equal

$$
\mathbb{Q}\left[\tau>T \mid \mathcal{G}_{t}\right]=\mathbb{E}^{\mathbb{Q}}\left[\exp \left(-\int_{t}^{T} \eta_{u}+J_{u} d u\right) \mid \mathcal{F}_{t}\right]=S_{\eta}(t, T) S_{J}(t, T)=S(t, T) .
$$

Defaultable bond prices under zero recovery. The price of zero coupon bond under zero recovery computes similarly. On $\{\tau>t\}$ it equals

$$
\bar{p}_{0}(t, T):=\mathbb{E}^{\mathbb{Q}}\left[\exp \left(-\int_{t}^{T} r_{u}+\eta_{u}+J_{u} d u\right) \mid \mathcal{F}_{t}\right]=\bar{S}_{\eta}(t, T) S_{J}(t, T)=\bar{S}(t, T) .
$$

Default digital payoffs. Evaluating a payment directly at $\tau$ typically involves computing

$$
e(t, T):=\mathbb{E}^{\mathbb{Q}}\left[\mu_{T} e^{-\int_{t}^{T} r_{u}+\mu_{u} d u} \mid \mathcal{F}_{t}\right]=\bar{\Gamma}(t, T)
$$

Note also that $e(t, T)=\mathbb{E}^{\mathbb{Q}}\left[\mu_{T} e^{-\int_{t}^{T} r_{u}+\mu_{u} d u} \mid \mathcal{G}_{t}\right]=\bar{p}_{0}(t, T) \overline{\mathbb{E}}^{T}\left[\mu_{T} \mid \mathcal{G}_{t}\right]$ where $\overline{\mathbb{E}}^{T}$ is the expectation under the $T$-survival measure. Furthermore, the expected value of the intensity under the $T$-survival measure computes to

$$
\overline{\mathbb{E}}^{T}\left(\mu_{T} \mid \mathcal{F}_{t}\right)=\frac{\bar{\Gamma}(t, T)}{\bar{p}_{0}(t, T)}=\frac{\bar{\Gamma}_{\eta}(t, T) S_{J}(t, T)+\Gamma_{J}(t, T) \bar{S}_{\eta}(t, T)}{\bar{S}_{\eta}(t, T) S_{j}(t, T)} .
$$

Recovery. With the survival probability at hand it is easy to consider bond prices under recovery of treasury or similar schemes. Here, we therefore concentrate on recovery of market value $(\mathrm{RMV})$. It is well known, that a $T$-defaultable asset $\mathcal{X}$ under RMV has the price

$$
\bar{\pi}_{R M V}(t)=\mathbf{1}_{\{\tau>t\}} \mathbb{E}^{\mathbb{Q}}\left[e^{-\int_{t}^{T} r_{s}+q \mu_{s} d s} \mathcal{X} \mid \mathcal{F}_{t}\right]+\mathbf{1}_{\{\tau \leq t\}} e^{\int_{\tau}^{t} r_{s} d s}(1-q) \bar{\pi}_{R M V}(\tau-),
$$


where $q$ is the loss quote and $\bar{\pi}_{R M V}(\tau-)$ is its pre-default value. The expectation equals

$$
\mathbb{E}^{\mathbb{Q}}\left[e^{-\int_{t}^{T} r_{s}+q \mu_{s} d s} \mid \mathcal{F}_{t}\right]=\bar{S}_{\eta}(q, t, T) S_{J}(q, t, T)=\bar{S}(q, t, T) .
$$

If we would like to include random recovery, independent of all the other factors, the result holds with $\mathbb{E}^{\mathbb{Q}}(q)$ instead of $q$.

As a next step we consider default digital puts (DDP) and credit default swaps (CDS). The CDS is the most liquid credit risky product, so pricing formulas are necessary for calibration to real data. For further examples we refer to Gaspar (2006).

Default Digital put. A DDP pays 1 directly at default if default happens before or at $T$. Its value at time $t$ (given no previous default) is

$$
\mathbb{E}^{\mathbb{Q}}\left[e^{-\int_{t}^{\tau} r_{u} d u} \mathbf{1}_{\{\tau<T\}} \mid \mathcal{G}_{t}\right]=\mathbb{E}^{\mathbb{Q}}\left[\int_{t}^{T} \exp \left(-\int_{t}^{s} r_{u}+\mu_{u} d u\right) \mu_{s} d s \mid \mathcal{F}_{t}\right]=\int_{t}^{T} \bar{\Gamma}(t, s) d s .
$$

Credit Default Swap. Entering a CDS obliges to the exchange of the following payments at the payment dates $T_{1}<T_{2}<\cdots<T_{N}$ :

- Fixed leg: pays $\bar{s} \cdot\left(T_{i}-T_{i-1}\right)$ if there was no default in $\left(T_{i-1}-T_{i}\right]$ (and previously)

- Floating leg: pays ${ }^{11}$ the difference between the nominal value and the recovery value if default occurred in $\left(T_{i-1}, T_{i}\right]$.

At the contract time $t$, the spread $\bar{s}(t)$ of the CDS is determined in such a way that the initial value of the CDS is zero. The spread remains fixed such that as time passes by the value of the CDS is typically not zero. For simplicity, we take the nominal value to be 1 . The value at time $t$ of the fixed leg is then $\bar{s} \sum_{i=1}^{N}\left(T_{i}-T_{i-1}\right) \bar{p}_{0}\left(t, T_{i}\right)$.

To compute the floating leg, we need the value of 1 unit of money payed at $T_{n}$ if default happens in $\left(T_{i-1}, T_{i}\right]$, which we denote by $e^{*}\left(t, T_{i-1}, T_{i}\right)$. Although $e^{*}\left(t, T_{i-1}, T_{i}\right)$ is not one of the building blocks, it is closely related to $e$ :

$$
e\left(t, T_{i}\right)=\lim _{T_{i-1} \rightarrow T_{i}} \frac{1}{T_{i}-T_{i-1}} e^{*}\left(t, T_{i-1}, T_{i}\right) .
$$

A generalization of Theorem 3.8 gives $e^{*}\left(t, T_{i-1}, T_{i}\right)$ explicitly (see Proposition A.1 in the appendix) and the credit spread $\bar{s}(t)$ becomes

$$
\bar{s}(t)=q\left(\sum_{i=1}^{N} e^{*}\left(t, T_{i-1}, T_{i}\right)\right)\left(\sum_{i=1}^{N}\left(T_{i}-T_{i-1}\right) \bar{p}_{0}\left(t, T_{i}\right)\right)^{-1} .
$$

\subsection{Portfolio credit risk}

In this section, we consider defaultable securities issued by several companies $k=1, \cdots, \bar{K}$. We assume that each company may default at most once and denote the default time of company $k$ by $\tau^{k}$. Set $\mathbf{k}=\{1, \cdots, \bar{K}\}$.

\footnotetext{
${ }^{11}$ Alternatively to paying the default payment at a time $T_{i}$ (and possibly including accrued interest) one may consider a payment directly at $\tau$. It is an easy exercise to compute this price using Theorem 3.8 .
} 
Assumption 4.1. Consider i.i.d. processes $\mu^{i}, i \in \mathbf{k} \cup\{c\}$, of the form quadratic ${ }^{12}$ plus jump, i.e. $\mu_{t}^{i}=\eta_{t}^{i}+J_{t}^{i}$ and

$$
J_{t}^{i}=\sum_{\tilde{\tau}_{j}^{i} \leq t} Y_{j}^{i} h^{i}\left(t-\tilde{\tau}_{j}^{i}\right), \quad \eta_{t}^{i}=Z_{t}^{\top} \mathrm{Q}^{i}(t) Z_{t}+\mathrm{g}^{i}(t)^{\top} Z_{t}+\mathrm{f}^{i}(t) .
$$

$\tau_{k}, k=1, \ldots, \bar{K}$ are doubly stochastic random times with intensities $\lambda^{k}=\mu^{k}+\epsilon^{k} \mu^{c}$ with $\epsilon^{k} \in \mathbb{R}_{+}$. Furthermore, the risk-free short rate $r$ is independent of any $\mu^{k}$ but not necessarily of the common intensity $\mu^{c}$.

Incorporating different factors for different sectors is straightforward. We note that in the multi-firm setup the building blocks will have firm-specific as well as common components. However, since firm-specific and common factors are, by definition, independent this does not represent an increase in computational difficulty. The next remark exemplifies, with survival probabilities, how easy it is.

Remark 4.2. The survival probability of company $k$ equals, on $\{\tau>t\}$,

$$
\begin{aligned}
& \mathbb{Q}\left(\tau>T \mid \mathcal{F}_{t}\right)=\mathbb{E}^{Q}\left(\exp \left[-\int_{t}^{T}\left(\mu_{s}^{k}+\epsilon^{k} \mu_{s}^{c}\right) d s\right] \mid \mathcal{F}_{t}\right) \\
& =\mathbb{E}^{Q}\left(\exp \left[-\int_{t}^{T} \mu_{s}^{k} d s\right] \mid \mathcal{F}_{t}\right) \mathbb{E}^{Q}\left(\exp \left[-\epsilon^{k} \int_{t}^{T} \mu_{s}^{c} d s\right] \mid \mathcal{F}_{t}\right)=S^{k}(t, T) S^{c}\left(\epsilon^{k}, t, T\right) .
\end{aligned}
$$

The higher $\epsilon^{k}$ the bigger is the dependence of company $k$ on the common default risk driver $\mu^{c}$. For intuition take $\epsilon^{k} \equiv \epsilon$. Then, if $\mu^{c}$ jumps, suddenly the default risk of all companies increases a lot and we will see numerous defaults. The nature of the shot noise process allows to pull back the intensity to usual levels quite fast, which will lead to clusters of defaults. This mimics contagion effects. On the other hand, an effect like this can also be caused by a rise in the quadratic part to a high level, but then it is more or less predictable in turn yielding something like a business cycle effect, such that on bad days more companies default than on good days.

Default Correlation. The quadratic-shot noise model offers more flexibility than Duffie and Gârleanu (2001). In affine model considered in Duffie and Gârleanu (2001) the meanreversion speed applies to the jump and to the diffusive part simultaneously, leading to either unrealistic high mean-reversion levels or to too low default correlations. In contrast, the quadratic-shot noise approach allows to address the mean reversion speed separately and leads to a better empirical fit. As will be shown in the next section, the proposed concrete model is able to produce realistically high default correlation for reasonable parameter values. The default correlation of name $i$ and $j$ is defined by $\rho^{i, j}(t, T):=\operatorname{corr}\left(\mathbf{1}_{\left\{\tau_{i} \leq T\right\}}, \mathbf{1}_{\left\{\tau_{j} \leq T\right\}} \mid \mathcal{F}_{t}\right)$. From Theorem 3.8 we obtain

$$
\rho^{i, j}(t, T)=\frac{\sqrt{S^{i}(t, T) S^{j}(t, T)}\left[S^{c}\left(\epsilon^{i}+\epsilon^{j}, t, T\right)-S^{c}\left(\epsilon^{i}, t, T\right) S^{c}\left(\epsilon^{j}, t, T\right)\right]}{\sqrt{\left(1-S_{D}^{i}(t, T)\right)\left(1-S^{j}(t, T)\right)}} .
$$

The pricing of portfolio credit derivatives as First-to-Default Swaps and CDOs, using the above setup, is treated in Gaspar and Schmidt (2008).

\footnotetext{
${ }^{12}$ We note that to get indepence of $\mu^{i}$ we also need, in particular, independence of $\eta^{i}$. Given that we are dealing with the same $Z$ state variables independence is achieved imposing, for a given $i$, that if we have $\left(Q^{i}\right)_{j} \neq 0$ or $\left(g^{i}\right)_{j} \neq 0$, then $\left(Q^{i}\right)_{j}=0,\left(g^{k}\right)_{j}=0$ for all $k \neq i$. In words, any element in $Z$ can only appear in one $\eta^{i}$.
} 
Calibration issues. Calibrating a portfolio of credit names to market data typically involves calibrating to single name derivatives as well as to portfolio products. Of course, it is possible to make a full calibration over all prices. However, it might be preferable to calibrate to single name derivatives first and in a second step fit to the portfolio products. For example, this allows to test different dependence scenarios, keeping the marginals fixed and changing the dependence structure. We therefore discuss in detail, how this can be achieved in our setting. A simple calculation shows that the factor approach is not suitable for this, as the nonlinearity in $D$ interferes with linear dependence on $\epsilon$ in the other term. As proposed already in Duffie and Gârleanu (2001), a way out is to consider $\lambda^{k}$ to be a sum of independent, but not identically distributed quadratic-shot noise models. The main tool is the following result, which is an extension of Proposition 1 in Duffie and Gârleanu (2001).

We say that the process $\mu$ is quadratic-shot noise with parameters $\left(\mathrm{Q}, \mathrm{g}, \mathrm{f}, l, h, F_{Y}\right)$ if it is as in Assumption 3.1; $F_{Y}$ denotes the distribution of $Y_{1}$.

Proposition 4.3. Consider two independent processes $\mu^{1}$ and $\mu^{2}$, both quadratic-shot noise with parameters $\left(\mathrm{Q}_{1}, \mathrm{~g}_{1}, \mathrm{f}_{1}, l_{1}, h, F_{Y_{1}}\right)$ and $\left(\mathrm{Q}_{2}, \mathrm{~g}_{2}, \mathrm{f}_{2}, l_{2}, h, F_{Y_{1}}\right)$, respectively. Set $q:=l_{1}\left(l_{2}+\right.$ $\left.l_{2}\right)^{-1}$. Then $\mu^{1}+\mu^{2}$ is also quadratic shot-noise with parameters $\left(\mathrm{Q}_{1}+\mathrm{Q}_{2}, \mathrm{~g}_{1}+\mathrm{g}_{2}, \mathrm{f}_{1}+\right.$ $\left.\mathrm{f}_{2}, l_{1}+l_{2}, h, q F_{Y_{1}}+(1-q) F_{Y_{2}}\right)$.

Proof. The result for the quadratic part immediately follows from (5). For the shot noise part, observe that the shot noise part of $\mu^{i}$ is $\sum_{j=1}^{N_{t}^{i}} Y_{j}^{i} h\left(t-\tau_{j}^{i}\right)$. Set $\tilde{J}_{t}:=\sum_{j=1}^{N_{t}^{1}} Y_{j}^{1} h(t-$ $\left.\tau_{j}^{1}\right)+\sum_{j=1}^{N_{t}^{2}} Y_{j}^{2} h\left(t-\tau_{j}^{2}\right)$. Then the jump times from $\tilde{J}$ have the same distribution as the jump times from a Poisson process with intensity $l_{1}+l_{2}$. Furthermore, the jumps have distribution $F_{Y_{1}}$ with probability $q$ and $F_{Y_{2}}$ with probability $1-q$, i.e. $\tilde{J}$ has the same distribution as a process $\sum_{j=1}^{N_{t}} Y_{j}\left(t-\tau_{j}\right)$, where $N$ is a Poisson process with intensity $l_{1}+l_{2}, Y_{i}$ are i.i.d. and $Q\left(Y_{1} \leq x\right)=q F_{Y_{1}}(x)+(1-q) F_{Y_{2}}(x)$.

With this result at hand, one can choose the parameters in such a way that the marginals are kept fixed and the dependence structure changes.

For a calibration of this model one therefore may interpolate (for each name) between every parameter except $h$. However, in typical cases one would rather leave Q, g, f as well as $F_{Y}$ untouched and vary $l_{i}$ to have the largest impact on the default correlation. To illustrate the concept, we give a more concrete example in the following section.

\section{A concrete model}

In this section we illustrate the results derived in the previous sections with a concrete three-factor model. Consider $Z=\left(Z^{1}, Z^{2}, r\right)^{\top}$ as the state variable with $\mathbb{Q}$-dynamics

$$
\begin{aligned}
d Z_{t}^{i} & =\left[\beta_{i}(t)-\alpha_{i} Z_{t}^{i}\right] d t+\sigma_{i} d W_{t}^{i}, \quad i=1,2 \\
d r_{t} & =\alpha_{r}\left[\beta_{r}-r_{t}\right] d t+\sigma_{r} \sqrt{r_{t}} d W_{t}^{r}
\end{aligned}
$$

where $\alpha_{i}, \sigma_{i}$, for $i=1,2, r$ and $\beta_{r}$ are constants, while $\beta_{i}(\cdot)$ are functions of $t$ and $W^{1}, W^{2}$ and $W^{r}$ are independent $\mathbb{Q}$-Wiener processes.

Factor approach. We will analyze two firms, denoted 1 and 2. Each firm's intensity is driven by firm-specific as well as common factors in accordance with Assumption 4.1. Consider $\epsilon^{1}, \epsilon^{2} \in \mathbb{R}$. The default intensity $\lambda^{k}$ of company $k, k=1,2$ is $\lambda_{t}^{k}=\mu_{t}^{k}+\epsilon^{k} \mu_{t}^{c}$ 
with $\mu_{t}^{k}=\eta_{t}^{k}=\left(Z_{t}^{k}\right)^{2}, \mu^{c}=J^{c}+\delta r$ and $J_{t}^{c}=\sum_{\tilde{\tau}_{i}<t} Y_{i} h\left(t-\tilde{\tau}_{i}\right)$, where $Y_{i} \sim \chi^{2}(2)$, $h(t)=e^{-b t}, b>0$ and $\tilde{\tau}_{i}$ are jumps of a Poisson process with intensity $l^{c}$.

Figure 3 shows simulated default times for different choices of $\epsilon_{i}$. The left plot has $\epsilon_{i}=0.1$ while the right plot has $\epsilon_{i}=0.5$. Especially the plot on the right hand side shows a strong dependence of the two default times which is due to the shot-noise part and mimics contagion effects.

We present all the necessary formulas and refer to Gaspar (2006) for full details. In the following we have $k=1,2$ and always set $x:=T-t$. We compute all building blocks:

- $S^{k}(\theta, t, T)=S_{\eta}^{k}(\theta, t, T)$ and

$$
\begin{aligned}
& S^{k}(\theta, t, T)=\sqrt{\frac{2 \gamma_{k} e^{\left(\gamma_{k}+\alpha_{k}\right) x}}{\left(\gamma_{k}+\alpha_{k}\right)\left(e^{2 \gamma_{k} x}-1\right)+2 \gamma_{k}}} \times \exp \left\{\frac{\theta\left[1-e^{2 \gamma_{k} x}\right]}{\left(\gamma_{k}+\alpha_{k}\right)\left[e^{2 \gamma_{k} x}-1\right]+2 \gamma_{k}}\left(Z_{t}^{k}\right)^{2}\right\} \\
& \quad \times \exp \left\{-\int_{t}^{T}\left(\beta_{k}(s) \mathcal{B}^{k}(\theta, s, T)+\frac{1}{2} \sigma_{k}^{2}\left(\mathcal{B}^{k}(\theta, s, T)\right)^{2}\right) d s+\mathcal{B}^{k}(\theta, t, T) Z_{t}^{k}\right\},
\end{aligned}
$$

where $\gamma_{k}=\sqrt{\alpha_{k}^{2}+2 \theta \sigma_{k}^{2}}$ and $\mathcal{B}^{k}$ is

$$
\begin{aligned}
& \mathcal{B}^{k}(t, T)=\left[2\left(\alpha_{k}+\gamma_{k}\right)\left(e^{2 \gamma_{k} x}-1\right)+4 \gamma_{k}\right]^{1 / 2 \sigma_{k}^{2}} \int_{t}^{T} \frac{4 \beta_{k}(s) \theta e^{\left(\alpha_{k}+\frac{\theta}{\gamma_{k}-\alpha_{k}}\right)(s-t)}\left(1-e^{2 \gamma_{k}(T-s)}\right)}{\left[2\left(\alpha_{k}+\gamma_{k}\right)\left(e^{2 \gamma_{k}(T-s)}-1\right)+4 \gamma_{k}\right]^{1+\frac{1}{2 \sigma_{k}^{2}}}} d s . \\
& \bullet S_{\eta}^{c}(\theta, t, T)=\left(\frac{2 \gamma_{\Delta} e^{\left(\gamma_{\Delta}+\alpha_{r}\right) \frac{x}{2}}}{\left(\alpha_{r}+\gamma_{\Delta}\right)\left[e^{\gamma_{\Delta} x}-1\right]+2 \gamma_{\Delta}}\right)^{\frac{2 \alpha_{r} \beta_{r}}{\sigma_{r}^{2}}} \exp \left\{\left(\frac{2 \Delta\left[1-e^{\gamma_{\Delta} x}\right]}{\left(\gamma_{\Delta}+\alpha_{r}\right)\left[e^{\gamma_{\Delta} x}-1\right]+2 \gamma_{\Delta}}\right) r_{t}\right\}
\end{aligned}
$$

where we set $\gamma_{\Delta}=\sqrt{\alpha_{r}^{2}+2 \sigma_{r}^{2} \Delta}$ and $\Delta=\theta \delta$. Similarly, $\bar{S}_{\eta}^{c}$ is obtained by replacing $\Delta$ by $\bar{\Delta}=(1+\theta \delta)$ in the above expression.

- For $S_{J}^{c}(\theta, t, T)$ we will make use of the Laplace transform of the $\chi^{2}(\nu)$ distribution $^{13}$ with $\nu=2 . D^{c}(\theta, s)=\frac{1}{b+2 \theta}\left[b+\frac{1}{s} \ln \left(1+\frac{2 \theta}{b}\left(1-e^{-b s}\right)\right)\right]$ and we find

$$
S_{J}^{c}(\theta, t, T)=\left[1+\frac{2 \theta}{b}\left(1-e^{-b x}\right)\right] \frac{l^{c}}{b+2 \theta} \exp \left\{\frac{J_{t}}{b}\left(e^{-b x}-1\right)-\frac{2 \theta x \cdot l^{c}}{b+2 \theta}\right\} .
$$

- $\Gamma^{k}(\theta, t, T)=\Gamma_{\eta}^{k}(\theta, t, T)=S_{\eta}^{k}(\theta, t, T) \exp \left(a^{k}(\theta, t, T)+b^{k}(\theta, t, T) Z_{t}^{k}+c^{k}(\theta, t, T)\left(Z_{t}^{k}\right)^{2}\right)$

with

$$
\begin{aligned}
a^{k}(\theta, t, T) & =-\int_{t}^{T} \beta_{k}(s)+\sigma_{k}^{2} \mathcal{B}^{k}(s, T) b_{k}+\sigma_{k}^{2} c^{k}(s, T) d s \\
b^{k}(\theta, t, T) & =-2 \int_{t}^{T} e^{\int_{t}^{s} \alpha_{k}-2 \sigma_{k}^{2} \mathcal{C}^{k}(u, T) d u}\left(\beta_{k}(s)-\sigma^{2} \mathcal{B}^{k}(s, T)\right) c^{k}(s, T) d s \\
c^{k}(\theta, t, T) & =\frac{\theta\left[\left(\gamma_{k}+\alpha_{k}\right)\left(e^{\gamma_{k} x}-1\right)+2 \gamma_{k}\right]}{\gamma_{k} e^{\left(\gamma_{k}+3 \alpha_{k}\right) x}},
\end{aligned}
$$

\footnotetext{
${ }^{13}$ Recall that for $u \geq 0$ the Laplace transform of random variable which has $\chi^{2}$ distribution with $\nu$ degrees of freedom, equals ${ }^{14}, \varphi_{\chi_{\nu}^{2}}(u)=\mathbb{E}\left(e^{-u \chi_{\nu}^{2}}\right)=(1+2 u)^{-\nu / 2}$.
} 

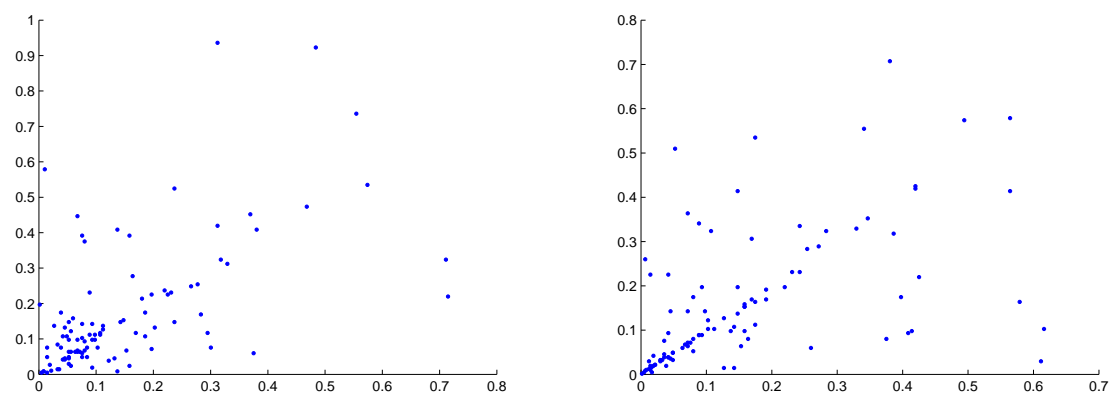

Figure 3: Simulated defaults of two companies according to the concrete model. To compare to copula simulations, the data is transformed to $[0,1]$ using the marginal distributions. Parameters are for $i=1,2: \beta_{i}=1, \alpha_{i}=0.5, \sigma_{i}=0.2, l^{c}=2, b=0.5$. $Y_{i} \sim \chi^{2}(2)$ and $r=0$. The left picture if for $\epsilon_{i}=0.1$, the right $\epsilon_{i}=0.5$.
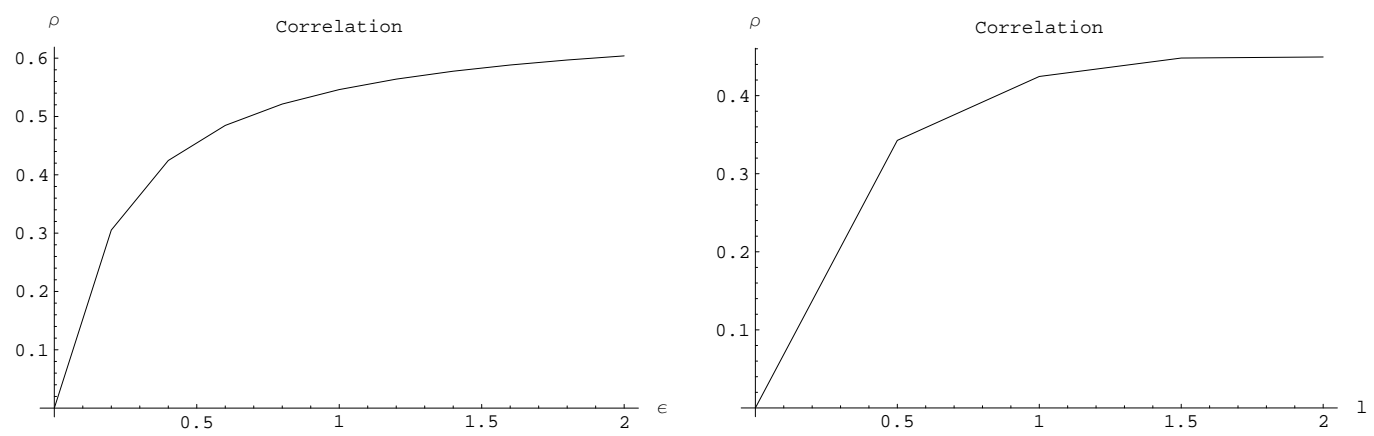

Figure 4: Model parameters: $\alpha=0.5, \beta=0.1 \alpha, \sigma=0.1, b=0.5$. The Graph shows the correlation for varying $\epsilon=\epsilon_{1}=\epsilon_{2}$ (left, $l^{c}=1$ ) and $l=l^{c}$ (right, $=\epsilon_{1}=\epsilon_{2}=0.4$ ).

$\mathcal{B}^{k}$ as above and $\mathcal{C}^{k}(\theta, t, T)=\theta\left[1-e^{2 \gamma_{k} x}\right] \cdot\left[\left(\gamma_{k}+\alpha_{k}\right)\left[e^{2 \gamma_{k} x}-1\right]+2 \gamma_{k}\right]^{-1}$.

- $\Gamma_{\eta}^{c}(\theta, t, T)=S_{\eta}^{c}(\theta, t, T) \exp \left\{a(\Delta, t, T)+\left(\frac{\theta\left[\left(\alpha_{r}+\gamma_{\Delta}\right)\left(e^{\gamma_{\Delta} x}-1\right)+2 \gamma_{\Delta}\right]}{2 \gamma_{\Delta} e^{\left(3 \alpha_{r}+\gamma_{\Delta}\right) \frac{x}{2}}}\right) r_{t}\right\}$, where $a(\Delta, t, T)=\int_{t}^{T} \alpha_{r} \beta_{r} b(\Delta, s, T) d s$. Recall that $\Delta=\theta \delta$ and $\bar{\Delta}=1+\theta \delta$. Similar as above, we obtain $\bar{\Gamma}_{\eta}^{c}$ replacing $\Delta$ by $\bar{\Delta}$.

- Finally, since $J^{c}(t, T)=\sum_{\tilde{\tau}_{i} \leq t} Y_{i} e^{-b\left(T-\tilde{\tau}_{i}\right)}$ we have that $\Gamma_{J}^{c}(\theta, t, T)$ equals $S_{J}^{c}(\theta, t, T)\left\{\theta J^{c}(t, T)-l^{c}\left[\frac{1}{2+b \theta}\left(b+\frac{1}{x} \ln \left(1+\frac{2 \theta}{c}\left(1-e^{-b x}\right)\right)(1-x)-1\right]+\frac{x}{1+\frac{2 \theta}{b}\left(1-e^{-b x}\right)}\right\}\right.$.

As mentioned before, these building blocks, now computed in closed-form (up to some numerical integrations) are sufficient to derive all relevant expressions for credit risk: survival probabilities, prices of credit derivatives, correlations, etc. In Figure 4 we use the above expressions and Equation (21) to illustrate the default correlation in this concrete model.

Separate calibration of marginals and dependence structure. Alternatively, the above formulas may be used to separately calibrate marginals and dependence structure, as mentioned previously. For ease of notation we consider $r=0$ and $l_{1}=l_{2}=l$. The default intensity is $\lambda_{t}^{k}=\mu_{t}^{1}+\mu_{t}^{2}$, where $\mu_{t}^{i}=\left(Z_{t}^{i}\right)^{2}+J_{t}^{c}+J_{t}^{i}$ are quadratic-shot noise, $J_{t}^{i}=\sum_{\tau_{j}^{i} \leq t} Y_{j}^{i} h\left(t-\tau_{j}^{i}\right), i=1,2, c$ are independent shot noise processes with intensities $l^{i}, i=1,2, c$. The fraction $l^{c} / l^{i}+l^{c}$ corresponds to the fraction of common jumps attributed to obligor $i$. The key building in this case are obtained similar as above. 


\section{A Appendix: an auxiliary result}

Proposition A.1. We have the following

$$
e^{*}\left(t, T_{n-1}, T_{n}\right)=\bar{p}_{o}\left(t, T_{n-1}\right) e^{\alpha\left(t, T_{n-1}, T_{n}\right)+\beta^{\top}\left(t, T_{n-1}, T_{n}\right) Z_{t}+Z_{t}^{\top} \gamma\left(t, T_{n-1}, T_{n}\right) Z_{t}}-\bar{p}_{o}\left(t, T_{n}\right),
$$

where $\alpha, \beta$ and $\gamma$ are deterministic functions and solve the following system of ODE

$$
\begin{aligned}
& \left\{\begin{aligned}
\frac{\partial \alpha}{\partial t}+d^{\top}(t) \beta+\frac{1}{2} \beta^{\top} k_{0}(t) \beta+\operatorname{tr} \gamma k_{0}(t)+\beta^{\top} k_{0}(t) \bar{B} & =0 \\
\alpha\left(T_{n-1}, T_{n-1}, T_{n}\right) & =A\left(T_{n-1}, T_{n}\right)
\end{aligned}\right. \\
& \left\{\begin{aligned}
\frac{\partial \beta}{\partial t}+E^{\top}(t) \beta+2 \gamma d(t)+\frac{1}{2} \tilde{\beta}^{\top} K(t) \beta+2 \gamma k_{0}(t) \beta & \\
+2 \bar{C} k_{0}(t) \beta+2 \gamma k_{0}(t) \bar{B}+\tilde{\beta}^{\top} K(t) \bar{B} & =0 \\
\beta\left(T_{n-1}, T_{n-1}, T_{n}\right) & =B\left(T_{n-1}, T_{n}\right)
\end{aligned}\right. \\
& \left\{\begin{aligned}
\frac{\partial \gamma}{\partial t}+\gamma E(t)+E^{\top}(t) \gamma+2 \gamma k_{0}(t) \gamma+\frac{1}{2} \tilde{\beta}^{\top} G(t) \tilde{\beta} & \\
+4 \bar{C} k_{0}(t) \gamma+\tilde{\bar{B}}^{\top} G(t) \tilde{\beta} & =0 \\
\gamma\left(T_{n-1}, T_{n-1}, T_{n}\right) & =C\left(T_{n-1}, T_{n}\right)
\end{aligned}\right.
\end{aligned}
$$

$A, B$ and $C$ are from Result 2.4, while $\bar{B}$ and $\bar{C}$ are from Theorem 3.8. $\alpha, \beta, \gamma$ should be evaluated at $\left(t, T_{n-1}, T_{n}\right)$ and $\bar{B}, \bar{C}$ at $\left(t, T_{n-1}\right)$.

Proof. The expected discounted value of 1 , payed at $T_{n}$, if $\tau \in\left(T_{n-1}, T_{n}\right]$ is

$$
\begin{aligned}
e^{*}\left(t, T_{n-1}, T_{n}\right) & =\mathbb{E}^{\mathbb{Q}}\left[e^{-\int_{t}^{T_{n}} r_{s} d s}\left(\mathbf{1}_{\left\{\tau>T_{n-1}\right\}}-\mathbf{1}_{\left\{\tau>T_{n}\right\}}\right) \mid \mathcal{G}_{t}\right] \\
& =\mathbb{E}^{\mathbb{Q}}\left[e^{-\int_{t}^{T_{n}} r_{s} d s-\int_{t}^{T_{n-1}} \mu_{s} d s} \mid \mathcal{F}_{t}\right]-\bar{p}_{0}\left(t, T_{n}\right) .
\end{aligned}
$$

Furthermore, for the remaining expectation we have that

$$
\begin{array}{r}
\mathbb{E}^{\mathbb{Q}}\left[e^{-\int_{t}^{T_{n}} r_{s} d s} e^{-\int_{t}^{T_{n-1}} \mu_{s} d s} \mid \mathcal{F}_{t}\right]=\mathbb{E}^{\mathbb{Q}}\left[e^{-\int_{t}^{T_{n-1}} r_{s}+\mu_{s} d s} p\left(T_{n-1}, T_{n}\right) \mid \mathcal{F}_{t}\right] \\
=\mathbb{E}^{\mathbb{Q}}\left[e^{-\int_{t}^{T_{n-1}} r_{s}+\eta_{s} d s} p\left(T_{n-1}, T_{n}\right) \mid \mathcal{F}_{t}^{W}\right] \mathbb{E}^{\mathbb{Q}}\left[e^{-\int_{t}^{T_{n-1}} J_{s} d s} \mid \mathcal{F}_{t}^{J}\right]
\end{array}
$$

because of the independence between $(J)$ and the other terms. The last expectation was computed in Theorem 3.8. So it remains to show that $\quad \mathbb{E}^{\mathbb{Q}}\left[e^{-\int_{t}^{T_{1}} r_{s}+\eta_{s} d s} p\left(T_{1}, T_{2}\right) \mid \mathcal{F}_{t}^{W}\right]=$ $=e^{\alpha\left(t, T_{1}, T_{2}\right)+\beta^{\top}\left(t, T_{1}, T_{2}\right) Z_{t}+Z_{t}^{\top} \gamma\left(t, T_{1}, T_{2}\right) Z_{t}} \cdot e^{\bar{A}\left(t, T_{1}\right)+\bar{B}^{\top}\left(t, T_{1}\right) Z_{t}+Z_{t}^{\top} \bar{C}\left(t, T_{1}\right) Z_{t}}$.

We imitate the proof of Lemma 3.6 and the notation from therein may be recalled. In particular, we need to specify $G$ and we use the ansatz $y\left(t, z, T_{1}\right)=g\left(t, z, T_{1}\right) e^{h\left(t, z, T_{1}\right)}$. From Result 2.4 we have that $p\left(T_{1}, T_{2}\right)=\exp \left(A\left(T_{1}, T_{2}\right)+B^{\top}\left(T_{1}, T_{2}\right) Z_{T_{1}}+Z_{T_{1}}^{\top} C\left(T_{1}, T_{2}\right)\right)$ thus, we set $G\left(T_{1}, Z_{T_{1}}, T_{2}\right)=p\left(T_{1}, T_{2}\right)$. We find that

$$
\left\{\begin{aligned}
\frac{\partial g}{\partial t}+\sum_{i} \frac{\partial g}{\partial z_{i}} \alpha_{i}+\frac{1}{2} \sum_{i j}\left(\frac{\partial^{2} g}{\partial z_{i} \partial z_{j}}+\frac{\partial g}{\partial z_{i}} \frac{\partial h}{\partial z_{j}}+\frac{\partial g}{\partial z_{j}} \frac{\partial h}{\partial z_{i}}\right) \sigma_{i} \sigma_{j} & =0 \\
g\left(T_{1}, z, T_{1}, T_{2}\right) & =G\left(T_{1}, Z_{T_{1}}, T_{2}\right) .
\end{aligned}\right.
$$

Finally, we note $g\left(T_{1}, z, T_{2}\right)=\exp \left(\alpha\left(T_{1}, T_{2}\right)+\beta^{\top}\left(T_{1}, T_{2}\right) Z_{t}+Z_{t}^{\top} \gamma\left(T_{1}, T_{2}\right) Z_{t}\right)$, $\frac{\partial g}{\partial t}=\left(\frac{\partial \alpha}{\partial t}+\frac{\partial \beta}{\partial t} z+z^{\top} \frac{\partial \gamma}{\partial t} z\right) g, \frac{\partial g}{\partial z_{i}}=\left(\beta_{i}+2 \gamma_{i} z\right) g, \frac{\partial^{2} g}{\partial z_{i} \partial z_{j}}=\left[2 \gamma_{i j}+\left(\beta_{i}+2 \gamma_{i} x\right)\left(\beta_{j}+2 \gamma_{j} x\right)\right] g$. Replacing these in the above PDE, as well as $\alpha_{i}$ and $\sigma_{i} \sigma_{j}^{\top}$ from (2), leaves us with a PDE which is separable and solves the required ODEs given in the proposition. 


\section{References}

Altmann, T., T. Schmidt, and W. Stute (2007). A shot noise model for financial assets. forthcoming in IJTAF.

Bielecki, T. and M. Rutkowski (2002). Credit Risk: Modeling, Valuation and Hedging. Springer Verlag. Berlin Heidelberg New York.

Chen, L., D. Filipović, and H. V. Poor (2004). Quadratic term structure models for riskfree and defaultable rates. Mathematical Finance 14(4), 515-536.

Collin-Dufresne, P., R. Goldstein, and J. Hugonnier (2004). A general formula for valuing defaultable securities. Econometrica 72, 1377-1407.

Dassios, A. and J. Jang (2003). Pricing of catastrophe reinsurance \& derivatives using the cox process with shot noise intensity. Finance and Stochastics 7(1), 73-95.

Davis, M. and V. Lo (2001). Infectious defaults. Quantitative Finance 1, 382-387.

Duffie, D. and Gârleanu (2001). Risk and valuation of collateralized debt obligations. Financial Analysts Journal 57(1), 41-59.

Filipović, D. (2002). Separable term structures and the maximal degree problem. Mathematical Finance 12(4), 341-349.

Frey, R., C. Prosdocimi, and W. J. Runggaldier (2007). Affine credit risk models under incomplete information. In S. W. J. Akahori, S. Ogawa (Ed.), Stochastic Processes and Applications to Mathematical Finance, pp. 97-113.

Frey, R. and W. Runggaldier (2006). Credit risk and incomplete information: a nonlinear filtering approach. preprint, University of Leipzig.

Gaspar, R. M. (2004). General quadratic term structures for bond, futures and forward prices. SSE/EFI Working paper Series in Economics and Finance, 559.

Gaspar, R. M. (2006). Credit Risk and Forward Price Models. Ph. D. thesis, Stockholm School of Economics.

Gaspar, R. M. and T. Schmidt (2008). On the pricing of cdos. In G. Gregoriou and P. U. Ali (Eds.), Credit Derivatives. Chapman Hall. forthcoming.

Johnson, N. L., S. Kotz, and N. Balakrishnan (1994). Continuous Univariate Distributions (2nd ed.), Volume 1. John Wiley \& Sons. New York.

Lando, D. (2004). Credit Risk Modeling: Theory and Applications. Princeton University Press. Princeton, New Jersey.

McNeil, A., R. Frey, and P. Embrechts (2005). Quantitative Risk Management: Concepts, Techniques and Tools. Princeton University Press.

Mortensen, A. (2006). Semi-analytical valuation of basket credit derivatives in intensitybased models. Journal of Derivatives 13(4).

Schmidt, T. and W. Stute (2004). Credit risk - a survey. Contemporary Mathematics 336, $75-115$.

Schönbucher, P. J. (2003). Credit derivatives pricing models - models, pricing and implementation. JWS. 